\title{
Sequential release of nanoparticle payloads from ultrasonically burstable capsules
}

\author{
Stephen Kennedy ${ }^{\mathrm{a}, \mathrm{b}, \uparrow}$, Jennifer Hu ${ }^{\mathrm{a}, \dagger}$, Cathal Kearney ${ }^{\mathrm{a}, \mathrm{c}}$, Hadas Skaat ${ }^{\mathrm{a}}$, Luo Gu ${ }^{\mathrm{a}}$, Marco Gentili ${ }^{\mathrm{a}}$, \\ Herman Vandenburgh ${ }^{\mathrm{d}}$, David Mooney ${ }^{\mathrm{a}, *}$ \\ ${ }^{a}$ Wyss Institute for Biologically Inspired Engineering, John A. Paulson School of Engineering and Applied Sciences, Harvard \\ University, Cambridge, MA 02138, USA \\ ${ }^{\mathrm{b}}$ Department of Electrical, Computer, and Biomedical Engineering, Department of Chemical Engineering, University of Rhode \\ Island, Kingston, RI 02881, USA \\ ${ }^{\mathrm{c}}$ Department of Anatomy, Tissue Engineering Research Group and Advanced Materials and Bioengineering Research Center, \\ Royal College of Surgeons in Ireland, Dublin, Ireland \\ ${ }^{\mathrm{d}}$ Department of Molecular Pharmacology, Physiology and Biotechnology, Department of Pathology and Laboratory Medicine, \\ Brown University, Providence, RI, 02912, USA \\ * Corresponding authors. Tel: (617) 384-9624. (D. Mooney). \\ E-mail address: mooneyd@ seas.harvard.edu (D. Mooney). \\ $\dagger$ Authors contributed equally to this work
}

Keywords: ultrasound response, drug delivery, biomaterials, controlled release

\section{A B S T R A C T}

In many biomedical contexts ranging from chemotherapy to tissue engineering, it is beneficial to sequentially present bioactive payloads. Explicit control over the timing and dose of these presentations is highly desirable. Here, we present a capsule-based delivery system capable of rapidly releasing multiple payloads in response to ultrasonic signals. In vitro, these alginate capsules exhibited excellent payload retention for up to 1 week when unstimulated and delivered their entire payloads when ultrasonically stimulated for 10 to $100 \mathrm{~s}$. Shorter exposures (10 s) were required to trigger delivery from capsules embedded in hydrogels placed in a tissue model and did not result in tissue heating or death of encapsulated cells. Different types of capsules were tuned to rupture in response to different ultrasonic stimuli, thus permitting the sequential, on-demand delivery of nanoparticle payloads. As a proof of concept, gold nanoparticles were decorated with bone morphogenetic protein- 2 to demonstrate the potential bioactivity of nanoparticle payloads. These nanoparticles were not cytotoxic and induced an osteogenic response in mouse mesenchymal stem cells. This system may enable researchers and physicians to remotely regulate the timing, dose, and sequence of drug delivery on-demand, with a wide range of clinical applications ranging from tissue engineering to cancer treatment.

\section{Introduction}

Biological and physiological systems conduct themselves with a high degree of temporal complexity. This often demands drug delivery strategies that are capable of directing biological processes with temporal nuance [1,2]. Furthermore, in certain instances, control over not only the timing but the sequence of biomolecular delivery is highly desired in order to mimic the manner by which nature regulates biological processes [3-5]. However, contemporary polymer-based

\section{$-1-$}


drug delivery materials that rely on diffusion or degradation typically provide either constant rates of release over protracted periods of time or predefined sequences of drugs [6,7].

Implantable materials that deliver payloads in response to external cues (i.e., $\mathrm{pH}$ [8], temperature [9], magnetic fields [10-12], electric fields [13,14], and optical signals [15]) provide a means by which the timing of different drug deliveries may be externally regulated [1]. External regulation of these drug deliveries provides the on-demand flexibility thought to be broadly required in a wide range of investigative and clinical scenarios [16].

Ultrasound is used in a wide range of biomedical contexts, can be administered to patients with a high degree of spatiotemporal precision $[17,18]$, and is being explored as a means to trigger the release of therapeutics from implantable polymeric systems. In these hydrogel systems, ultrasonic disruption of the polymer and/or the polymer cross-links enables the release of incorporated therapeutic payloads [17,19-21]. These ultrasonically responsive systems therefore have tremendous potential in a wide range of applications that require localized and temporal control over drug delivery. However, there are several issues with ultrasonically responsive systems that currently limit their use in certain applications. First, triggered deliveries from these systems rely on disruptive forces that must be minimized. For instance, in tissue engineering applications it is highly desirable to trigger therapeutic deliveries at lower energies in order to minimize the risk of damaging nearby and nascent tissues, preserve the structural integrity of implanted scaffolding materials, and preserve the conformation and bioactivity of delivered therapeutics. Second, many ultrasonically responsive systems lack adequate payload retention when unstimulated. Thus, demonstrations of delayed release after days to weeks are currently lacking even though some applications may require delayed deliveries over these timescales. For example, in bone regeneration applications, 1-week delayed delivery of bone morphogenetic protein-2 (BMP-2) (subsequent to initial inflammatory and angiogenic stages) may be beneficial [5,23-25]. Finally, the ability to sequentially trigger multiple payloads from ultrasonically responsive system has not yet been demonstrated, despite its need in many biomedical applications. For example, sequential delivery of pro-angiogenic and pro-maturation factors is thought to enhance the development of large and mature vasculature $[3,4]$. In cancer treatments, it is thought that the efficacy of chemotherapeutics can be enhanced by first delivering agents that make cancer cells more vulnerable to the chemotherapeutic [26]. For instance, it has been suggested that sequential 5-fluorouracil (5FU) and irinoteacan deliveries may enhance the toxicity of 5FU [27].

Taking these limitations and demands as motivation, we endeavored to develop polymeric capsules that possess several attributes that are desirable in a broad range of investigative and clinical scenarios. More specifically, these capsules were designed to retain nanoparticle payloads for up to a week and release their payloads at rates dictated by the intensity and duration of applied ultrasonic signals. Importantly, the ultrasonic signals required to trigger these releases were intended to not cause appreciable heating or damage to cells and tissues. These capsules were also designed to provide sequentially triggered payload deliveries. That is, capsules could be engineered with different susceptibilities to ultrasonic rupture (i.e., weaker versus stronger capsules made by using different types and amounts of cross-linker). Thus, systems integrated with both strong and weak capsules could be triggered to first release payloads contained in weaker capsules without triggering release from stronger capsules. More intense ultrasonic exposures can be used to subsequently trigger release of payloads contained in stronger capsules. The capsule-based materials described in this work were made from ionically cross-linked alginate, whose properties are conducive to a wide variety of biomedical 
applications as it is non-toxic, minimally immunogenic, and can be modified in a manner that permits tunable biodegradability [28]. We therefore believe that these capsules will be highly useful in a variety of applications that require flexible, on-demand control over the timing, dose, and sequence of multiple therapeutic payload deliveries.

\section{Materials and methods}

\subsection{Synthesis and decoration of gold nanoparticles}

Gold nanoparticles (AuNPs) were synthesized via citrate reduction as described in previous work [29]. $0.4 \mathrm{~L}$ of $98.5 \mu \mathrm{g} / \mathrm{mL}$ gold (III) chloride trihydrate (Sigma Aldrich, St. Louis, MO) was magnetically stirred and heated to a rolling boil. $10 \mathrm{~mL}$ of $7.5 \mathrm{mg} / \mathrm{mL}$ sodium citrate (Sigma Aldrich) solution was rapidly added, leading to the formation of AuNPs of roughly $25 \mathrm{~nm}$ diameter. The solution was allowed to cool at room temperature. Deionized water was added to a final volume of $0.4 \mathrm{~L}$. To decorate AuNPs with bone morphogenetic protein-2 (BMP-2), we adopted a similar strategy as described in Kim et al. [28] for decorating AuNPs with a different growth factor-vascular endothelial growth factor (VEGF). $1 \mu \mathrm{g}$ of recombinant human BMP-2 (R\&D Systems, Minneapolis, MN) was diluted in $120 \mu \mathrm{L}$ of deionized water and added in $40 \mu \mathrm{L}$ increments to $120 \mu \mathrm{L}$ stock AuNP solution with 20-minute room temperature incubations on a rocker between additions. The solution was then left overnight on the rocker at room temperature, followed by four centrifugation-washes at $13000 \mathrm{RCF}$ for $20 \mathrm{~min}$ to remove remaining unbound BMP-2. To produce PEGylated AuNPs in control experiments, AuNPs were incubated with $10 \mathrm{~mL} 0.1 \mathrm{mM} 2 \mathrm{kDa}$ thiol-mPEG (JenKem Technology USA, Plano, TX) at room temperature overnight, stirring, followed by four centrifugation-washes at 13,000 RCF for 20 min each to remove unreacted PEG.

\subsection{Synthesis of alginate capsules}

In order to make ultrasonically burstable capsules, cross-linker solutions were prepared from particles (AuNPs as described above or in some cases $<5 \mu \mathrm{m}$ iron (II, III) oxide microparticles (Sigma Aldrich)), divalent cation (either calcium chloride or barium chloride (Sigma Aldrich)), and $100 \%$ w/v sucrose (Sigma Aldrich), stored at $4{ }^{\circ} \mathrm{C}$. A glass dish was filled to a depth of $2 \mathrm{~cm}$ with $0.25 \mathrm{wt} \%$ LF 20/40 alginate solution (FMC BioPolymer, Philadelphia, PA). An automatic syringe pump holding a 5-mL syringe fitted with an 18-gauge needle was fixed above the dish such that the distance between the needle and the surface of the alginate bath was $2 \mathrm{~cm}$. The syringe pump allowed $250 \mu \mathrm{l}$ of cross-linker solution to drip at a flow rate of $1 \mathrm{~mL} / \mathrm{min}$ into the alginate bath, where the divalent cation in the cross-linker solution quickly formed a capsule around the payload. After gelation times of 3 to 10 minutes, completed capsules were briefly rinsed in deionized water and stored in phosphate-buffered saline (PBS) (Sigma). Images of capsules filled with iron oxide micro-particles were taken on a stereoscopic dissection microscope at $4 \times$ magnification, and ImageJ was used to quantify capsule diameters. To compare the force at failure of different capsules, individual capsules were compressed at a uniform strain rate of $2 \mathrm{~mm} / \mathrm{min}$ on an Instron model 3345 (Instron, Norwood, MA) until rupture. 


\subsection{Incorporation of capsules in bulk hydrogel structures}

In order to incorporate ultrasonically responsive capsules in bulk hydrogel structures, calcium-cross-linked alginate gels were cast around the capsules. $4 \mathrm{~mL}$ of $2.5 \mathrm{wt} \%$ alginate dissolved in 4-morpholineethanesulfonic acid (MES) buffer (Sigma Aldrich) was mixed with $900 \mu \mathrm{L}$ of MES buffer and $100 \mu \mathrm{L} \mathrm{CaSO}_{4}$ slurry $\left(210 \mathrm{mg} / \mathrm{mL}\right.$ of $\mathrm{CaSO}_{4}$ dihydrate (Sigma Aldrich) in deionized water). This mixing was accomplished quickly by linking two 5-mL syringes together with a LuerLock connector (W. W. Granger, Inc., Lake Forest, IL) and ejecting the mixture into custom cylindrical, 9-mm-diameter Teflon molds prefilled with capsules. These capsule-incorporated gels were allowed to set for 30 minutes and then stored in PBS. The resulting gels contained $2 \mathrm{wt} \%$ alginate with a calcium cross-linking concentration of $5 \mathrm{mM}$.

\subsection{Ultrasonic exposure experiments}

Capsules were exposed to ultrasonic energy in a number of manners. In all studies, a Vibra-Cell VCX 130 sonicator (Sonics \& Materials, Inc., Newtown, CT) was used with a 13-mm probe (Sonics, Newton, CT). The ultrasonic signals used here were administered to samples at various input amplitudes $(20 \%, 40 \%$, and $80 \%)$ as indicated in the readout on the Vibra-Cell VCX 130's display. Measurements indicating the ultrasonic intensities associated with these amplitude percentages are included in Appendix A: Supplementary Data. For experiments where capsules were exposed to ultrasound while suspended in solution, a total of $250 \mu \mathrm{l}$ of AuNP-containing capsules ( 20 capsules, each containing AuNP concentrations of $211.2 \mu \mathrm{g} / \mathrm{mL}$ ) were placed in a $50 \mathrm{~mL}$ conical tube with $5 \mathrm{~mL}$ of PBS. The sonicator was positioned in the center of the tube $2 \mathrm{~cm}$ from the bottom and delivered a range of ultrasonic doses. Doses were defined by two metrics: ultrasound amplitude ( $20 \%$ to $80 \%$ as read from the sonicator's display) and duration ( 1 to $500 \mathrm{~s}$ ). All ultrasonic exposures used $20 \mathrm{kHz}$ signals. Immediately after sonication, a sample of solution was collected and its AuNP concentration was quantified by optical absorption $(518 \mathrm{~nm})$ with a plate reader (BioTek Instruments, Inc., Winooski, VT) against a standard of known AuNP concentrations.

For experiments using chicken carcasses, capsules containing iron oxide micro-particles or bulk gels containing two capsules each were placed under the skin of chicken wings, medial to the ulna, that were warmed in an incubator to $37^{\circ} \mathrm{C}$. The sonicator probe was coated with Aquasonic 100 ultrasound-conducting gel (Parker Laboratories, Inc., Fairfield, NJ) and lowered such that the gel bridged the gap between the sonicator probe and the tissue surface. Ultrasound was applied at $20 \%$ or $80 \%$ amplitude for ten seconds. The temperature of the treated tissue (the surface of the skin facing the gel) was measured by a thermocouple probe immediately before and after sonication. Iron oxide microparticles were incorporated into capsules in these studies so that ultrasonic rupture was visually apparent.

\subsection{Nanoparticle toxicity and bioactivity studies}

Clonally derived mouse bone marrow stromal mesenchymal stem cells (D1 mMSCs, ATTC, Manassas, VA) - which have previously been used in osteogenesis studies [30] - were used in all viability studies and bioactivity studies. mMSCs were seeded at 10,000 cells $/ \mathrm{cm}^{2}$ and cultured in standard Dulbecco's Modified Eagles Medium (DMEM, Invitrogen, Carlsbad, CA) with $10 \%$ fetal bovine serum and $1 \%$ penicillin/streptomycin (Invitrogen), at $37^{\circ} \mathrm{C}$ and $5 \% \mathrm{CO}_{2}$. 
During experiments, media was refreshed every three days. mMSC viability was quantified via live-dead staining (Invitrogen) after 7 days of exposure to AuNPs and controls. Representative images from an epifluorescent microscope were processed using Image J software in order to quantify viability percentages. In experiments where the cell count and viability of mMSCs were quantified after having been encapsulated in bulk alginate hydrogels, alginate gels were dissolved by submerging them in a solution of $50 \mathrm{mM}$ ethylene-diaminetetraacetic acid (EDTA, Sigma Aldrich) in PBS for 10 minutes at $37^{\circ} \mathrm{C}$. mMSCs were then collected and analyzed for cell count (cell counter, Beckman Coulter, Inc., Pasadena, CA) and viability (live-dead staining, Invitrogen).

mMSC differentiation was measured in order to demonstrate the bioactivity of BMP-2decorated AuNPs. In these differentiation studies osteogenic-supplemented medium (osteoDMEM) was used that consisted of DMEM supplemented with $50 \mu \mathrm{g} \mathrm{mL}^{-1} \mathrm{~L}$-ascorbic acid and $10 \mathrm{mM} \beta$-glycerophosphate (Sigma Aldrich). Differentiation of mMSCs was measured by the activity of alkaline phosphatase (ALP) in cells seeded in a 48-well plate at 10,000 cells/well and cultured for 1 week. $10 \mu \mathrm{l}$ of 4-methylumbelliferyl phosphate disodium salt (MUP, Sigma Aldrich) was added to each well and incubated for 25 minutes at $37^{\circ} \mathrm{C}$. ALP was quantified with a plate reader (Biotek, excitation/emission $=360 \mathrm{~nm} / 440 \mathrm{~nm}$ ) using bovine ALP (Sigma Aldrich) to generate a standard curve. The ALP activity per well was normalized to mMSC cell count in each well.

\subsection{Data representation and statistical analyses}

All quantitative data were represented as means \pm standard deviation. The majority of statistical comparisons were limited to a single comparison. For these single comparisons, a student t-test was applied to calculate significance with $p$-values of less than 0.05 being the benchmark for significance. For instances where multiple comparisons were made across groups (i.e., Fig. 4 D), one-way Analysis of Variance (ANOVA) with a Tukey's post hoc test was utilized. Throughout this work, unless otherwise stated, the following convention was used to indicate the level of significance: $* * *$, and $* * *$ indicate $p<0.05,0.01$, and 0.001 , respectively. "n.s." indicates that no statistical significance was found.

\section{Results and discussion}

\subsection{Ultrasonically responsive capsule fabrication and characterization}

Alginate capsules were designed to rapidly rupture in response to ultrasonic signals. A solution containing divalent cations (i.e., calcium or barium chloride), sucrose (to make the droplets less buoyant when placed in the alginate bath), and payloads (i.e., gold nanoparticles or iron oxide microparticles) was added dropwise to an alginate bath (Fig. 1 A(i)). Once in the bath, these droplets released their divalent cations to the surrounding polymer solution (Fig. 1 A(ii)) eventually forming a cross-linked alginate network as a capsule wall (Fig. 1A (iii)). This process resulted in alginate capsules that encapsulated deliverable payloads (Fig. 1 A(iv), here containing dark iron oxide microparticles for higher visual contrast). These capsules were roughly $4 \mathrm{~mm}$ in diameter with $0.5 \mathrm{~mm}$ walls (Fig. $1 \mathrm{~B}$ ). These capsules were capable of 
releasing iron oxide microparticles in a matter of seconds when ultrasonically stimulated (Fig. 1 C).
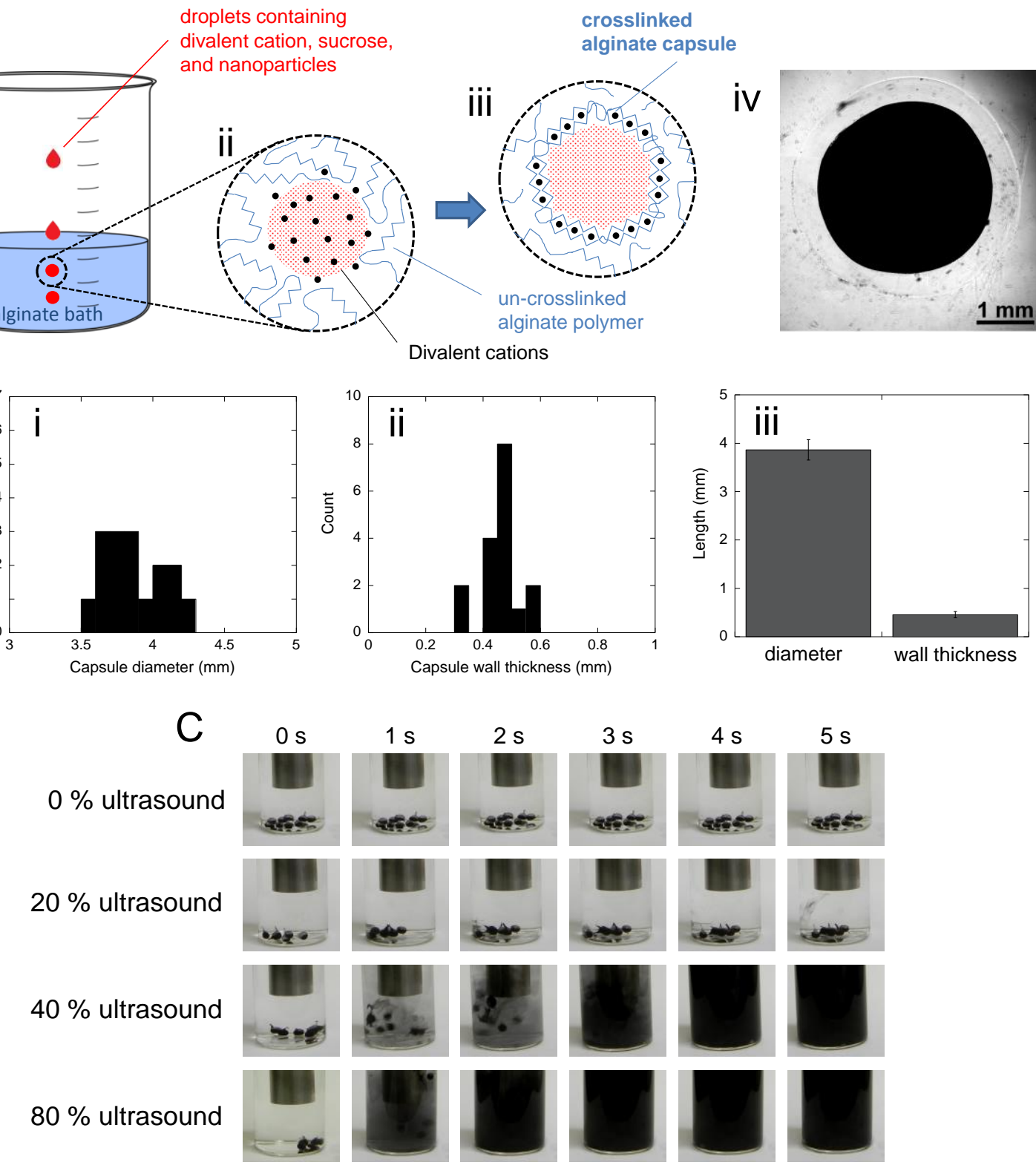

Fig. 1. Alginate capsules can be fabricated to rupture in response to ultrasound. (A) Schematic of the capsule fabrication process: (i) droplets containing divalent cations, sucrose, and nanoparticle payloads are added drop-wise to an alginate bath, (ii) once submerged, these droplets release divalent cations which interact with the alginate, (iii) eventually forming an ionically cross-linked capsule wall. (iv) A representative microscopic image of a capsule loaded with iron oxide particles formed using $50 \mathrm{mM} \mathrm{CaCl}_{2}$. (B) Histograms of capsule diameter and wall thickness (i,ii) distributions and (iii) comparisons of mean values \pm standard deviations $(\mathrm{N}=8)$. (C) Images of $10 \mathrm{~mL}$ PBS solutions containing iron-oxide-loaded capsules when subjected to the indicated ultrasonic signals for 5 seconds. These experiments were conducted in glass scintillation vials with the $13-\mathrm{mm}$ ultrasound probe placed $2 \mathrm{~cm}$ above the capsules. 


\section{2. $\quad$ Triggered delivery of gold nanoparticles}

In order to characterize the payload retention and ultrasonically stimulated release capabilities of these capsules, they were loaded with gold nanoparticles (Fig. 2 A, inset) and stimulated with ultrasound after being submerged in PBS for 7 days. Capsules released low percentages of their payloads over the course of 7 days when not ultrasonically stimulated (Fig. 2 A and B). When ultrasonically stimulated on day 7 at $20 \%$ amplitude for 100 s, gold nanoparticle release rapidly transitioned from $1.3 \%$ to $100 \%$ release (Fig. 2 A). This corresponded to a $\sim 400,000$-fold increase in release rate from $3.4 \times 10^{-5}$ to $17.5 \mu \mathrm{g} / \mathrm{min}$ (Fig. $2 \mathrm{~B}$ ). These data demonstrate the ability to retain molecular payloads for days and deliver them rapidly in an ondemand manner. We attribute these capsules' excellent nanoparticle retention capabilities to the inability of nanoparticles to traverse the capsule wall. The gold nanoparticles used here were greater than $10 \mathrm{~nm}$ while the pore size of the alginate hydrogel comprising the capsule walls was on the scale of single-digit nanometers [28]. While several ultrasonically responsive drug delivery systems have recently reported excellent retention capabilities, our system's stimulated delivery over unstimulated delivery ( 400,000-fold vs. 5000- [22] and 14-fold [31] enhancements) and prolonged retention (7 days (or 168 hours) vs. $30 \mathrm{~s}$ [32] and $30 \mathrm{~min}$ [33]) represent considerable improvements. The timescales of delayed delivery afforded by these capsules (several days to a week) may provide improvements in tissue engineering strategies over typical delivery systems that provide initial burst release profiles [34] or sustained release $[6,7]$.

Capsules were also exposed to ultrasonic signals of various intensities and durations in order to quantify the relationship between ultrasonic stimulus and the amount of payload released. For example, when exposed to ultrasound at $20 \%$ amplitude, ultrasonic exposures of roughly 10 to 100 seconds corresponded to the transition from $0 \%$ to $100 \%$ nanoparticle release (Fig. 2 C, black curve). At higher ultrasonic percent amplitudes, this transition required shorter ultrasonic exposures (Fig. 2 C, blue and red curves). This demonstrates that not only the timing but the amount of payload delivered can be explicitly and remotely regulated using ultrasonic stimuli. While this regulatory capability has been demonstrated in other ultrasonically responsive hydrogel [35] and alginate capsule systems [36], the work presented here is notable in the use of relatively ephemeral ultrasonic exposures. The capsules presented here exhibit $100 \%$ payload release in only 10 to 100 seconds (depending on the ultrasonic amplitude) using ultrasonic signals applied in a wide range of other delivery modalities [19-22,31-40].

In practical scenarios requiring prolonged retention followed by triggered release, it was a concern that these capsules' retention and regulated release characteristics would change after being submerged in physiological media for several days. Specifically, the calcium cross-links in the alginate gel could be replaced by other ions in physiological media (e.g., $\mathrm{Na}^{+}$) over time. However, after being submerged in calcium-free PBS for a week, the same ultrasonic durations engendered statistically indistinguishable percentages of nanoparticle releases (Fig. 2 D, comparing solid and dashed curves). 

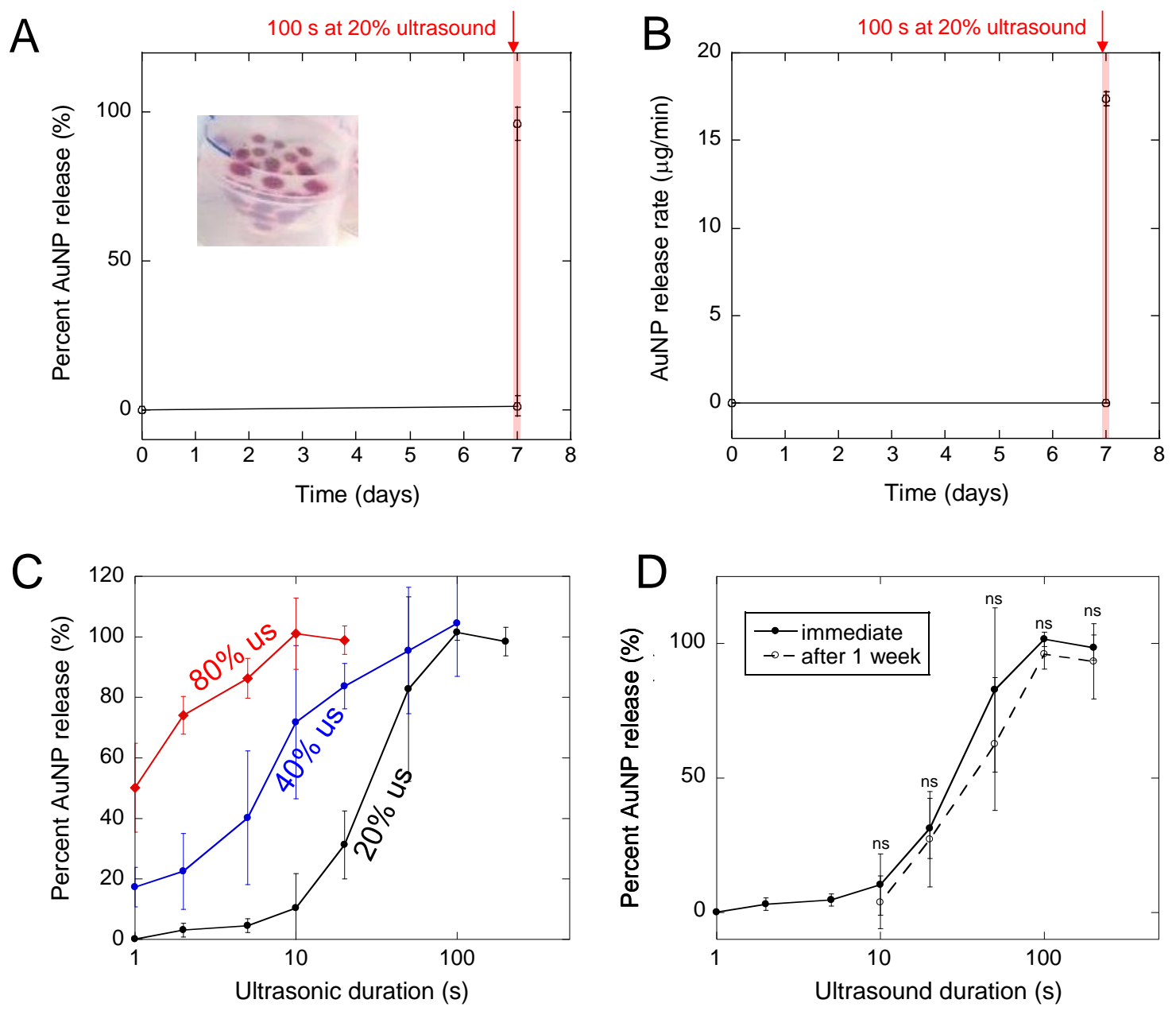

Fig. 2. Capsules retain their nanoparticle payloads for prolonged periods and can be ultrasonically triggered to release their payloads. (A) Percent release over time from gold-nanoparticle-loaded capsules (inset) that were exposed to ultrasound on day 7 for $100 \mathrm{~s}$ at $20 \%$ ultrasound. (B) For the same experiment as presented in part A, the rate of nanoparticle release over time. In parts $\mathrm{A}$ and $\mathrm{B}$, the red shaded region at day 7 indicates the time when capsules were exposed to ultrasound. (C) The percent release as a function of the duration of ultrasonic exposure for capsules exposed to 20\% (black), $40 \%$ (blue) and $80 \%$ (red) amplitudes. Note that the $x$-axis is log-scale. (D)

Percent release as a function of the ultrasound's duration when exposed to $20 \%$ ultrasound for capsules immediately after fabrication (solid curve) compared to capsules that sat in PBS for a week (dashed curve). For parts A-D, $\mathrm{N}=4$. In each experiment (A-D), capsules were placed in $50 \mathrm{~mL}$ tubes containing $5 \mathrm{~mL}$ of PBS with the sonicator's probe being centered azimuthally and placed $2 \mathrm{~cm}$ above the bottom of the tube.

\subsection{Sequential delivery of payloads}

In order to produce capsules that could be ultrasonically ruptured, one after the other, capsules were designed with varying susceptibilities to ultrasonic rupture. This was achieved by creating capsules with different amounts and types of cross-linkers. Capsules made from droplets containing $50 \mathrm{mM} \mathrm{CaCl}_{2}$ (Fig. $3 \mathrm{~A}$ (i) and Fig. $2 \mathrm{~A}$ (iv)) and those containing $100 \mathrm{mM}$ $\mathrm{BaCl}_{2}$ (Fig. 3A(ii)) exhibited similar inner diameters but statistically different wall thickness and 

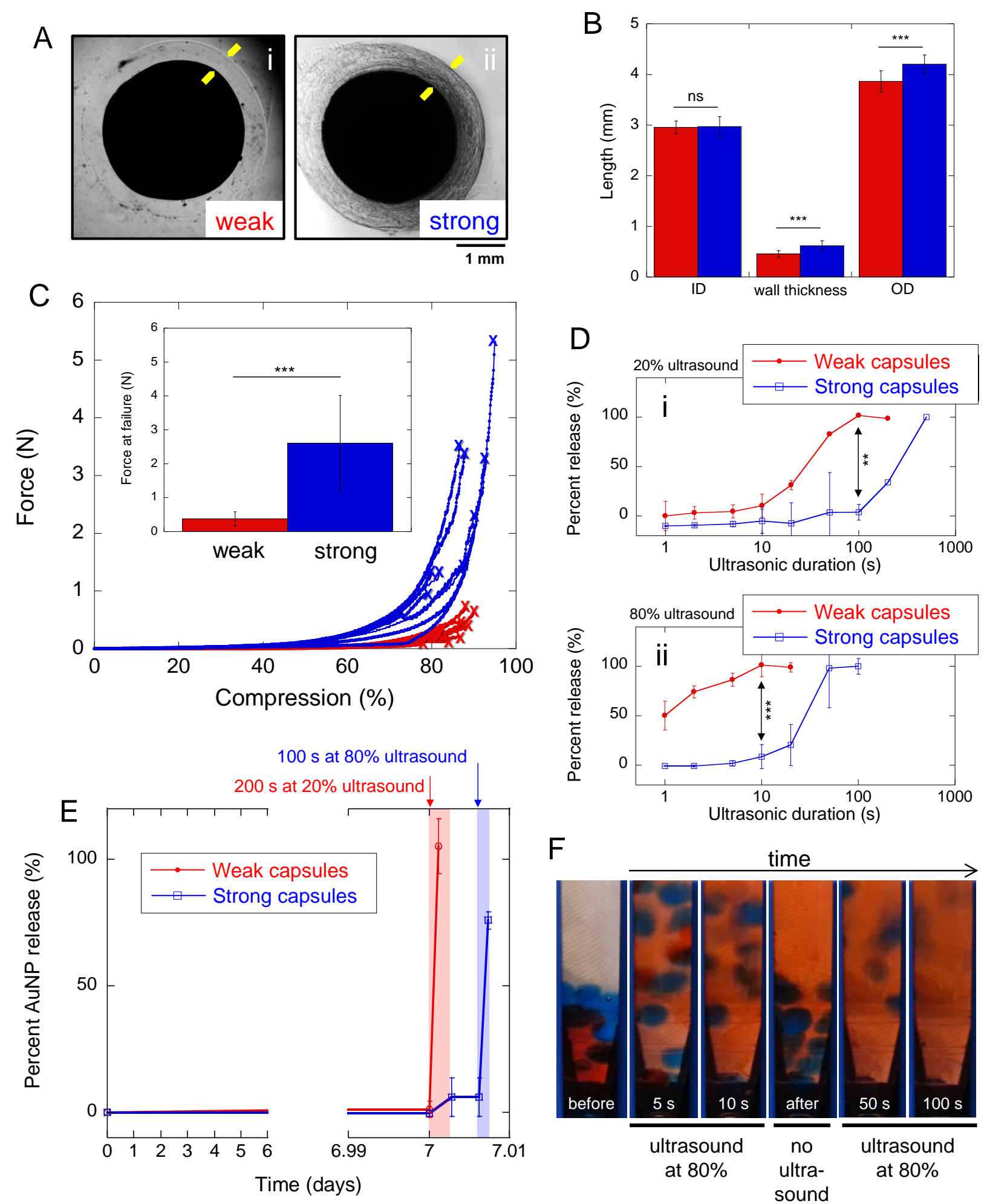

Fig. 3. Capsules can be engineered with varying strength and susceptibility to ultrasonic rupture to enable sequentially triggered payload deliveries. (A) Microscopic images of (i) weak capsules made by using $50 \mathrm{mM}$ $\mathrm{CaCl}_{2}$ and (ii) strong capsules made by using $100 \mathrm{mM} \mathrm{BaCl}_{2}$. Yellow arrows draw attention to the capsule wall boundaries. (B) Comparison of weak (red) and strong (blue) capsules' inner diameter (ID), capsule wall thickness, and outer diameter (OD). $\mathrm{N}=17-24$. (C) Applied force as capsules were compressed at $2 \mathrm{~mm} / \mathrm{min}$ until failure 
(indicated by ' $x$ ') for weak (red) and strong (blue) capsules. Inset: mean \pm standard deviations of the forces required to rupture weak and strong capsules under compression. $\mathrm{N}=8$. (D) The percent of gold nanoparticles released from strong and weak capsules as a function of ultrasonic exposure duration when exposed to (i) $20 \%$ and (ii) $80 \%$ ultrasound amplitudes. (E) Percent gold nanoparticle release over time for when both weak and strong capsules were exposed to $200 \mathrm{~s}$ of ultrasound on day 7 at $20 \%$ amplitude followed by $100 \mathrm{~s}$ of ultrasound five minutes later at $80 \%$ amplitude. For parts D and E, N = 4. (F) Images of weak (red) and strong (blue) capsules as they were exposed to ultrasound at $80 \%$ amplitude for long enough to rupture the weak capsules $(10 \mathrm{~s})$, followed by a pause ("no ultrasound"), and then an additional ultrasound to rupture the strong capsules (for an 100 additional seconds). Weak and strong capsules were loaded with red and blue food coloring, respectively.

outer diameters, by design (Fig. 3 B, comparing red and blue bars). The forces required to rupture these thicker-walled "strong" capsules were statistically higher than those required to rupture the thinner-walled "weak" capsules (Fig. 3 C, comparing blue data to red data). Aside from the difference in capsule wall thickness, we attributed this enhancement in capsule strength to the barium cross-linker, which has been shown to create stronger gels [41]. When both weak (red) and strong (blue) capsules were exposed to ultrasound at 20\% amplitude for 100s, 100\% nanoparticle release was observed in the weak capsules while nearly $0 \%$ release was observed in the strong capsules (Fig. 3 D(i)). A similar trend was observed when exposing both weak and strong capsules to ultrasound at $80 \%$ amplitude, where only $10 \mathrm{~s}$ of exposure preferentially triggered nanoparticle release from the weak capsules (Fig. $3 \mathrm{D}(\mathrm{ii})$ ). This ability to trigger delivery from weak capsules preferentially over stronger capsules enables sequential payload deliveries. For example, weak capsules retained their payloads for 7 days (Fig. 3 E). On day 7, a $20 \%$ amplitude ultrasonic signal could be used to trigger delivery from weak capsules with only a statistically insignificant amount of release from the strong capsules (Fig. 3 E, blue and red curves just after day 7). After ultrasound was terminated no additional release from the strong capsules was observed until an $80 \%$ amplitude ultrasonic signal was administered (Fig. 3 E, blue curve at day 7.01). Thus, payloads from the weak capsules were triggered first, followed by triggered delivery from the strong capsules at a later time (i.e., sequential delivery). Sequential delivery was also demonstrated by first triggering the delivery of red dye from weak capsules without triggering delivery from the strong capsules (Fig. $3 \mathrm{~F}$, at time points earlier than $10 \mathrm{~s}$ ). After this initial ultrasound was terminated, strong capsules remained un-ruptured (Fig. $3 \mathrm{~F}$, "after" photograph). When a longer ultrasonic signal was subsequently administered, strong capsules ruptured, releasing their payload (Fig. 3F, at time points later than $50 \mathrm{~s}$ ). Thus, these capsules enabled on-demand, sequentially triggered delivery of two different payloads.

To the best of our knowledge, this is the first demonstration of a tuned hydrogel system that is capable of being remotely triggered to release two payloads, in sequence, in response to independent ultrasonic signals. Elsewhere, others have demonstrated sequential release potential by tuning capsule architecture to provide different diffusive release rates [42] and by tuning capsule composition to have differential release responses when exposed to other types of triggering stimuli (i.e., when exposed to oil [43], piperidine or $\mathrm{HCl}$ [44]). Noble and coworkers [31] demonstrated that hydrogels could be coated differently to endow them with different ultrasonically triggered release characteristics. This hydrogel system exhibited excellent retention when unstimulated and differential release characteristics at various ultrasonic intensities based on hydrogel coatings. Lattin and Pitt [32] explored several factors influencing the potential tunability of ultrasonically responsive perfluorocarbon-loaded liposomes, including liposome size, the type of perfluorocarbon used in the liposomes, the ultrasonic intensity, and the ultrasonic frequency. These studies revealed several instances of preferential delivery when 
ultrasonically triggered due to liposomal tuning. We have also previously demonstrated that payload size can be tuned to enhance independently triggered control over ultrasonic deliveries [22]. While none these previous works explicitly demonstrated sequential delivery vs. time per se [22,31,32,43-44], taken along with the current study, these studies demonstrate that there are many design strategies one could adopt to generate flexible delivery profiles from these types of biomaterial systems.

\subsection{Decoration of gold nanoparticles with bioactive protein}

As a proof of concept, it was sought to demonstrate that gold nanoparticles potentially encased in these capsules could be decorated with protein and be used to viably induce a biological response. Due to the potential utility of these capsules in delaying deliveries in bone regeneration applications, mouse mesenchymal stem cells (mMSCs) were used here to characterize nanoparticle bioactivity. Despite the wealth of research on gold nanoparticles, their biological effects, and their application in clinical trials $[45,46]$, gold nanoparticles are not, in general, nontoxic, as toxicity depends on their specific functionalization and synthesis [47]. Therefore, the impact of these particles on mMSC viability was analyzed at the maximum possible gold nanoparticle concentration cells might experience upon capsule release (i.e., the nanoparticle concentration contained in these capsules: $211.2 \mu \mathrm{g} / \mathrm{ml})$. PEGylated gold nanoparticles at $211.2 \mu \mathrm{g} / \mathrm{ml}$ resulted in statistically similar mMSC viability levels as did controls at $0 \mu \mathrm{g} / \mathrm{ml}$ (Fig. $4 \mathrm{~A}$ ). In order to create particles with bioactive effects on mMSCs, thiol- or disulfide-gold interactions were used to decorate these gold nanoparticles with BMP2-a protein known to differentiate mMSCs down the osteogenic lineage [23]. BMP-2, which contains several cysteine residues (Fig. 4 B(i)) and disulfide bonds (Fig. 4 B(ii)), can interact with and bind to gold (Fig. 4 B(iii)) in a semi-covalent manner [22,48]. This principle has previously been applied to decorate gold nanoparticles with other similarly sized proteins while preserving the protein's bioactivity [29]. The BMP-2 on the gold-nanoparticle conjugates could be detected in a dose-dependent manner using enzyme-linked immunosorbent assay (ELISA) (Fig. 4 C). Effective concentrations of BMP-2 were calculated using ELISA and it was determined that $2.62 \mu \mathrm{g} / \mathrm{mL}$ of decorated AuNPs was equivalent to $300 \mathrm{ng} / \mathrm{mL}$ of unconjugated BMP-2. Note that the optical absorbance associated with the gold nanoparticles alone did not have detectible influence on the ELISA results unless using relatively high gold nanoparticle concentrations (Fig. 4 C, gray bars).

In order to further verify bioactivity, mMSCs were exposed to these BMP-2-goldnanoparticle conjugates and measured for ALP activity, a measure of osteogenic differentiation. It was found that exposure of these BMP-2-gold-nanparticle conjugates to mMSCs significantly enhanced ALP activity as compared to controls. $2.62 \mu \mathrm{g} / \mathrm{ml}$ of BMP-2-gold-nanoparticle conjugates (with an effective concentration of $300 \mathrm{ng} / \mathrm{mL}$ of BMP-2 measured by ELISA) resulted in similar enhancements in ALP activity as $300 \mathrm{ng} / \mathrm{mL}$ of BMP-2 alone (Fig. $4 \mathrm{D}$, comparing "AuNP-BMP" to "300 ng/mL BMP”). Both these conditions led to statistically significant enhancements in ALP activity as compared to controls (Fig. 4 D, comparing "AuNPBMP" and "300 ng/mL BMP" to "DMEM" and "osteo-DMEM"). Both BMP-2-decorated nanoparticles and $300 \mathrm{ng} / \mathrm{mL}$ of BMP-2 also led to statistically significant enhancements in ALP activity as compared to $10 \mathrm{ng} / \mathrm{mL}$ of BMP-2. By contrast, the ALP-inducing activity of PEGylated gold nanoparticles at $2.62 \mu \mathrm{g} / \mathrm{ml}$ was statistically indistinguishable from controls, indicating that gold nanoparticles themselves were not primarily responsible for ALP 
enhancement. It has been reported elsewhere, however, that PEGylated AuNPs can enhance MSC osteogenic differentiation [22,49].

A i
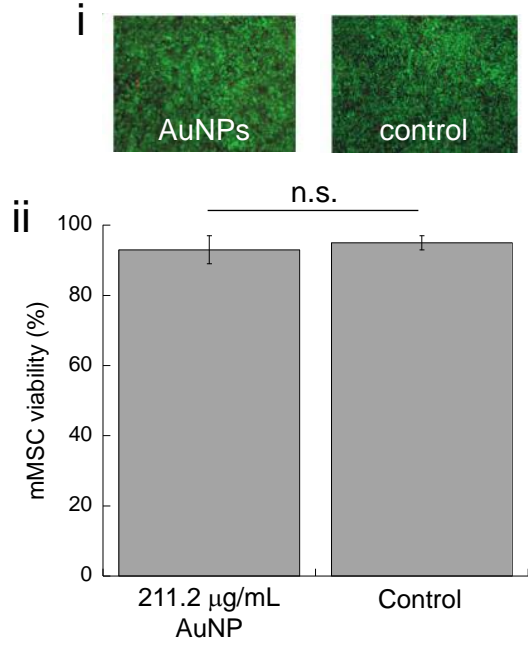

C

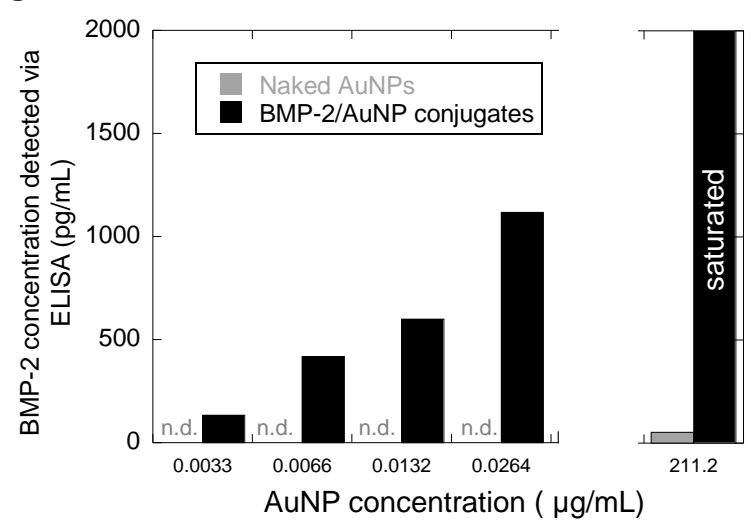

B

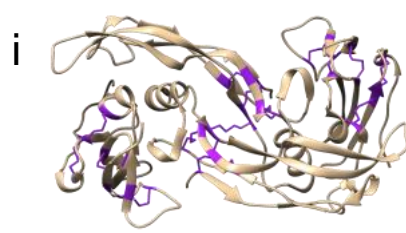

Cysteine residues

on BMP-2

ii<smiles></smiles>

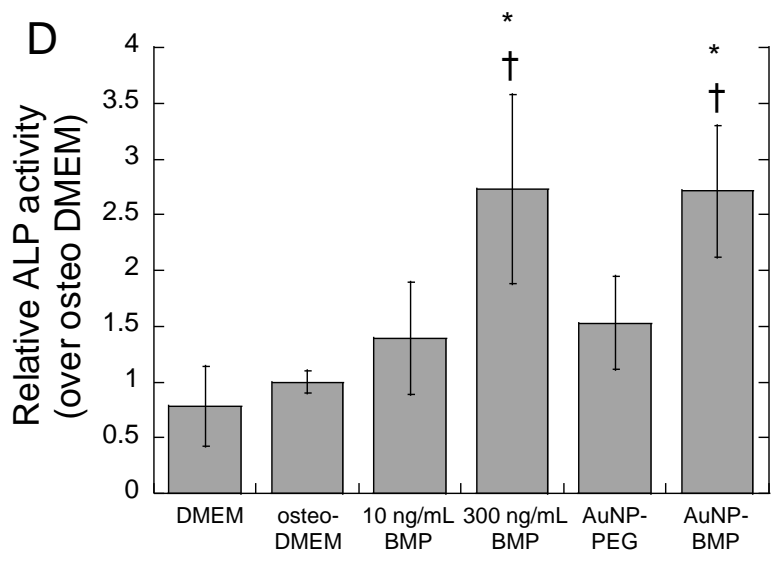

Fig. 4. The gold nanoparticles loaded into these ultrasonically responsive capsules can be endowed with osteogenic bioactivity. (A) (i) Images of live/dead stained mMSCs after exposure to $211.2 \mu \mathrm{g} / \mathrm{mL}$ of gold nanoparticles in DMEM for 7 days compared to a control with no nanoparticles. (ii) Quantitation of mMSC viability when exposed to gold nanoparticles and controls $(\mathrm{N}=4)$. (B) (i) BMP-2 includes several cysteine residues (ii) which contain disulfide bonds which can bind to gold substrates. (C) Detected BMP-2 concentrations when assaying the indicated concentrations of undecorated gold nanoparticles (gray) and BMP-2-decorated particles (black). n.d. indicates that no protein was detected using ELISA. The $211.2 \mu \mathrm{g} / \mathrm{mL}$ condition saturated the assay when using BMP-2-decorated nanoparticles. (D) Relative ALP activity (normalized to osteogenically supplemented media) for mMSCs after 7 days when exposed to the following: moving from left to right, normal culture media (DMEM), osteogenic supplemented cell media (osteo-DMEM), osteo-DMEM with 10 and $300 \mathrm{ng} / \mathrm{mL}$ of BMP-2, osteo-DMEM with 2.62 $\mu \mathrm{g} / \mathrm{mL}$ of PEG-decorated gold nanoparticles, and osteo-DMEM with $2.62 \mu \mathrm{g} / \mathrm{mL}$ of BMP-2-decorated gold nanoparticles (estimated to be equivalent to $300 \mathrm{ng} / \mathrm{mL}$ of BMP-2 when using ELISA) $(\mathrm{N}=3-4)$. * indicates statistically significant differences when compared to both DMEM and osteo-DMEM conditions $(\mathrm{p}<0.01)$ and $\dagger$ indicates statistically significant differences when compared to the $10 \mathrm{ng} / \mathrm{mL} \mathrm{BMP}-2$ condition $(\mathrm{p}<0.05)$. 


\subsection{Incorporation of capsules in bulk hydrogels}

For use in certain situations, it may be necessary to ultrasonically trigger these capsules while incorporated into hydrogel scaffolds that have been placed in tissues. Both weak and strong capsules were loaded with iron oxide microparticles (in order to easily visualize capsule rupture), incorporated into $2 \mathrm{wt} \%$ alginate gels that were cross-linked with $5 \mathrm{mM}$ calcium sulfate, and these constructs were placed under the skin of chicken carcasses (Fig. 5 A, left side of figure). After being ultrasonically stimulated at $20 \%$ amplitude for $10 \mathrm{~s}$ through the skin, weak capsules burst, releasing their iron oxide payloads (Fig. 5 A, image in top row under "After $20 \%$ ultrasound for 10 s"). Note that these ruptured weak capsules were not subsequently exposed to an $80 \%$ ultrasound signal because they had already ruptured. Strong capsules did not release their iron oxide payloads when exposed to this dose of ultrasound (Fig. 5 A, image in middle row under "After $20 \%$ ultrasound for 10 s"). However, when subsequently stimulated at a higher ultrasonic intensity, strong capsules released their payloads (Fig. 5 A, image in middle row under "After $80 \%$ ultrasound for 10 s"). When weak and strong capsules were integrated into the same alginate hydrogel, they were still capable of being sequentially triggered for release (Fig. 5 A, bottom row). That is, when exposed to the more moderate ultrasonic signal, the weak capsule in the gel ruptured and the strong capsule did not (Fig. 5 A, bottom row, second image from the right). At a later time, when a stronger ultrasonic signal was applied, the strong capsule ruptured (Fig. $5 \mathrm{~A}$, bottom row, rightmost image). Note that based on visual inspection the bulk hydrogel remained intact after ultrasonic treatment, which would be critical in tissue engineering applications requiring a scaffolding material that promotes cell adhesion, proliferation, and remodeling [50]. Also note that these ultrasonic signals required here were lower than those required to trigger release in experiments where capsules were suspended in PBS (Fig. 3 D). We attributed this to two factors. One, the ultrasonic probe is closer to the capsules when stimulating the implanted gel compared to when stimulated in PBS (a few millimeters as compared to $2 \mathrm{~cm}$ ). Two, when immobilized in a gel and/or a tissue, the ultrasonic energy is allowed to more consistently affect the area of the capsule most proximal to the ultrasonic probe, making the capsule rupture more efficiently. When submerged in PBS, these capsules tend to bounce around and rotate (Fig. 3 F), making ultrasonic rupture less efficient.

In examining another practical consideration, we aimed to determine if the ultrasonic energies required to trigger release heated tissues or influenced the viability of cells encapsulated within hydrogel scaffolding structures. When measured, tissue temperatures were similar before and after exposure to the ultrasonic does ( $80 \%$ amplitude for $10 \mathrm{~s})$ required to trigger release for strong capsules embedded in hydrogels structures inside our tissue model (Fig. 5 B).

Additionally, when mMSCs were encapsulated in bulk alginate hydrogels and exposed to the same ultrasonic signal $(80 \%$ amplitude for $10 \mathrm{~s})$, their viability and numbers did not decrease in a statistically significant manner (Fig. $5 \mathrm{C}$, viability in black and cell count in grey). While not statistically significant, we attributed the slight reduction in cell count (Fig. 5 C, grey bars) to cells lost on the perimeter of the gel, which are more likely to be affected by the ultrasonic signal. Nonetheless, a strong population of live cells remain in the gel after ultrasonic exposure $(\sim 350,000$ live cells per $0.5 \mathrm{~mL}$ gel). Note that the relatively low cell viabilities measured here were likely due to these alginate gels not being optimized for mMSC encapsulation (i.e., 2 wt \% alginate cross-linked with $5 \mathrm{mM}$ calcium sulfate and lacking RGD functionalization for proper cell adhesion). The maintenance of cell viability and tissue temperatures observed here are consistent with other reports in that the ultrasonic exposures utilized here are well below those 
determined to be harmful to cells [51,52]. Finally, in other more sensitive applications, improved targeting of these capsules can potentially be achieved through the use of high intensity focused ultrasound [53,54].
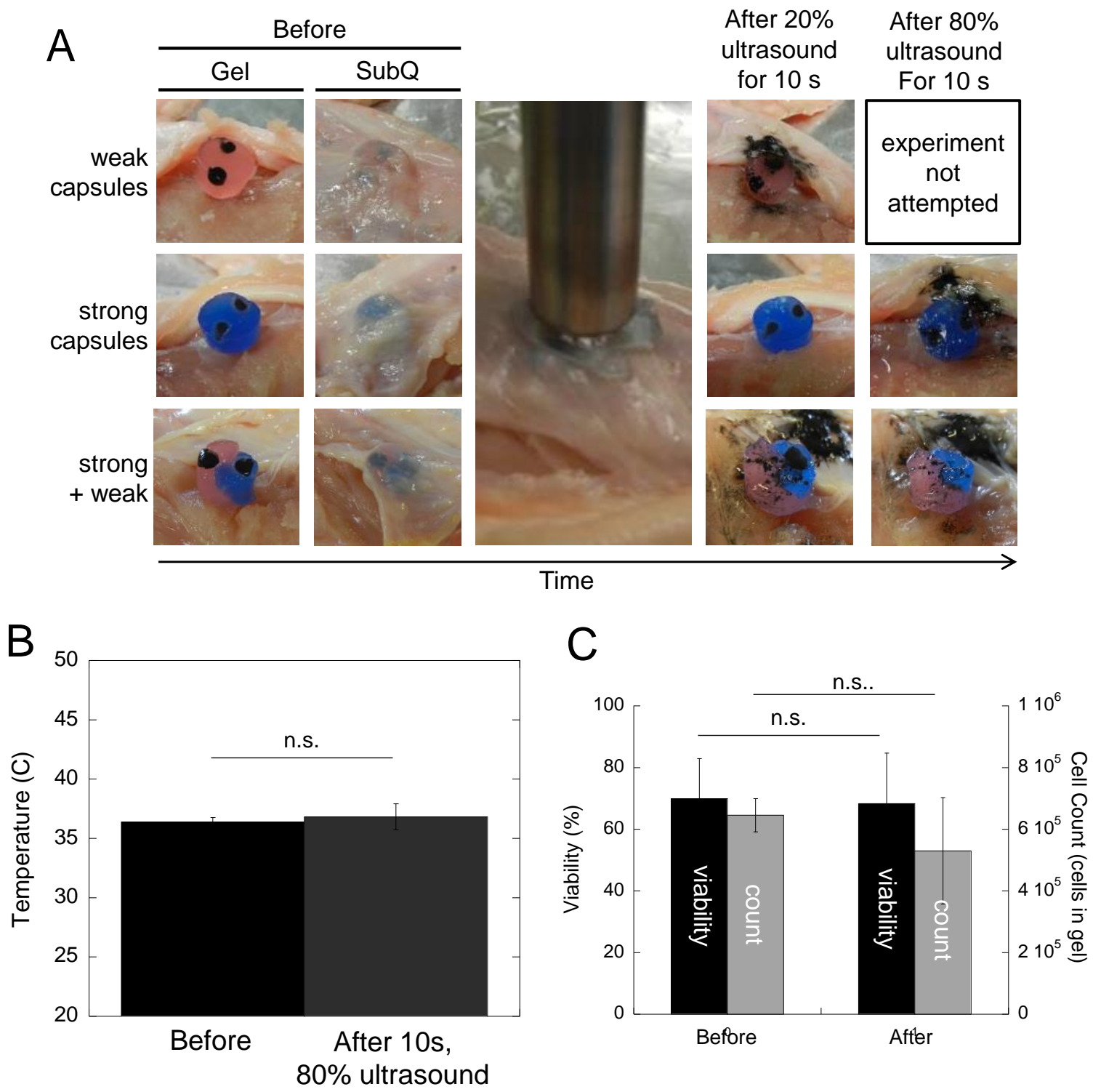

Fig. 5. Capsules can be ruptured when integrated into hydrogels and placed in a chicken carcass tissue model using ultrasonic signals that do not result in tissue heating or reductions in cell viability. (A) Images of iron-oxide-loaded capsules integrated into $2 \mathrm{wt} \%$ alginate hydrogels that are cross-linked with $5 \mathrm{mM}$ calcium sulfate before, during, and after ultrasonic stimulation when implanted under the skin of $37^{\circ} \mathrm{C}$ chicken carcass. The time over the course of each experiment moves from left to right. Note that gels containing only weak capsules (top row) were not stimulated at $80 \%$ ultrasound since all capsules ruptured using $20 \%$ ultrasound. Therefore, an image was not acquired for the $80 \%$ case. (B) Tissue temperature before and after exposure to 10 seconds of ultrasound at $80 \%$ amplitude. (C) Cell viably (black, left axis) and cell count (grey, right axis) of mMSCs encapsulated in 2 wt \% alginate hydrogels that are cross-linked with $5 \mathrm{mM}$ calcium sulfate and exposed to 10 seconds of ultrasound at $80 \%$ amplitude $(\mathrm{N}=4)$. 


\subsection{Applications, limitations, and future directions}

The capsule-based system presented here provides several notable advantages over previous work that make it a potentially useful tool for several applications, including bone tissue engineering applications. First, because the nanoparticle payloads are too large to diffuse out of the hydrogel capsules' walls, these capsules exhibit excellent payload retention (i.e., 1.3\% payload release over 7 days, or $0.0078 \%$ per hour, see Fig. 2 A). Second, when stimulated, these capsules (by design) rupture very rapidly, providing $100 \%$ payload release in an efficient manner ( $100 \%$ payload release in $<100 \mathrm{~s}$ of stimulation, or $1 \%$ per second). In comparison to this lab's previous work [19], these capsules' 78 -fold enhancement in payload retention rate $(0.0078 \% / \mathrm{h}$ compared to $\sim 0.6 \% / \mathrm{h})$ and 730 -fold enhancement in stimulated release rate $(1 \% / \mathrm{s}$ compared to $0.00137 \% / \mathrm{s}$ ), enabled the triggered release of payloads efficiently after prolonged periods of time (Fig 2 A and B, 7 day retention and rapid 100\% release on day 7). Due to these enhancements in both retention and release efficiency, when stimulated, these capsules provided a 400,000-fold enhancement in payload release rate over unstimulated release rate, compared to the previous system's 7-fold enhancement. Finally, because these capsules could be "tuned" to rupture at varying degrees of ultrasonic stimulation (Fig. 3 A through D, strong vs. weak capsules), payloads could be triggered to release in sequence (Fig. $3 \mathrm{~F}$, first triggered release of red payloads from weak capsules followed by a later triggering of blue payloads from strong capsules). This sequenced rupturing of weak then strong capsules was also demonstrated in a tissue model (Fig. $5 \mathrm{~A}$ ). The previous system was limited to releasing single payloads. In bone regeneration, it has been suggested that delaying the delivery of molecules that differentiate MSCs into osteoblasts can enhance regenerative outcome [5,23-25]. This system may be useful in these strategies since it was shown here that gold nanoparticles could both (i) be functionalized with BMP-2 and have a osteo-differentiating effect on MSCs (Fig 4 C and D) and (ii) could be retained for at least a week in these capsule systems (Fig. 2 A and B).

Several other treatment strategies could also benefit from a biomaterial system with the above-described capabilities. For example, in cancer treatment, it has been demonstrated that the pulsatile delivery profiles that these capsule-based systems can provide result in more efficient cancer cell destruction than the mostly constant delivery profiles provided by biomaterials that rely on diffusion and/or degradation [19]. This capsule system's specific ability to sequentially deliver one payload followed by a different payload could also provide further advancements in cancer treatment. For instance, in emerging anticancer strategies, more resistant cancer cells can be rendered less resistant by exposing them to a secondary agent [26]. However, selecting the right timing and sequence [27] of these independent deliveries will be critical in optimizing these clinical strategies. The material system described here may be useful in experimentally determining these optimized timings and sequences. In vaccine therapies, many important immunizations require several boosters in sequence following initial inoculation [55]. Several microsphere-based systems have been developed for single-administration vaccines [56-59]. The system described here could provide flexibility over the timing of these boosts and enable longer sequences of boosts.

However, before these capsule-based systems can be utilized in these and other biomedical contexts, several future studies will be required. In bone regeneration applications, the osteo-differentiating capacity of BMP/AuNP constructs must be verified before and after ultrasonically stimulated release. In cancer therapy applications, AuNPs that are functionalized with anticancer agents must be developed and the therapeutic impact of these AuNP constructs 
must be verified before and after sonication. Similarly, for vaccine inoculation and boosting, AuNPs must be created that are functionalized with antigen and adjuvants and verified to retain bioactivity before and after ultrasonically triggered release. Importantly, in both these and other applications, experiments must be conducted in live animal models. While the studies described here have demonstrated that these capsules can be ultrasonically ruptured in a tissue model, future studies will be required to fully characterize these system's capabilities in live animals. Specifically, though it was demonstrated here that AuNP delivery can be triggered after a week in vitro, demonstrating triggered delivery after prolonged implantation in live animals will be a critical step in applying these materials in therapeutic strategies. In these applications, it is of note that alginate in pure forms is well-known to cause minimal inflammation when implanted [28].

\section{Conclusions}

We have created alginate capsules that can deliver nanoparticle payloads upon application of ultrasonic stimuli. On-demand deliveries after storage times of up to one week in vitro were demonstrated, even in calcium-free buffer. The enhancement in release rate with stimulation was $\sim 400,000$-fold, as compared to the unstimulated release rate. Additionally, the amount of release could be regulated by the intensity and duration of the applied ultrasonic signal. We have also demonstrated that release from these capsules can be tuned by controlling the amount and type of cross-linker in the capsule walls. This enabled sequential payload delivery by first rupturing weakly cross-linked capsules, followed by more strongly cross-linked capsules. When integrated into bulk alginate hydrogel scaffolds and placed under the skin in a chicken carcass tissue model, these capsules retained their ability to be sequentially ruptured using ultrasonic stimuli. Payload deliveries from these capsules were rapid: $100 \%$ release when ultrasonically stimulated in under $100 \mathrm{~s}$ in vitro. However, when triggering release from capsules that were integrated in alginate scaffolds and placed in a chicken carcass tissue model, less than $10 \mathrm{~s}$ of ultrasound was required. This amount of ultrasonic stimulation did not result in measurable increases in tissue temperature or death of mMSCs encapsulated in the scaffolds. We believe that these demonstrated capabilities will be of great investigative value for examining how the timing, sequence, and dose of various biomolecules influence outcomes in cancer and tissue engineering research. We also believe that the on-demand capabilities that these capsules provide will have strong clinical applicability in situations where the timing, dose, and sequence of delivery must be modified in real time according to current patient prognoses.

\section{Acknowledgments}

The authors would like to thank Dr. Darinka Klumpers, Brian Kwee, and Ting-Yu Shih for help with mMSC experiments. J.H. thanks the staff of Engineering Sciences 100 at Harvard University for their guidance and patience during the portions of this work associated with her senior design project. This work was supported by a grant from the National Institutes of Health (NIH) (2 R01 DE013349). S.K.'s contributions were partially supported by start-up funds from the College of Engineering at the University of Rhode Island, an Early Career Development Award from the Rhode Island IDeA Network for Biomedical Research Excellence (RI-INBRE, 
NIH National Institute of General Medical Sciences, 2 P20 GM103430), a Medical Research Grant from the Rhode Island Foundation (20144262), and an EPSCoR Track II grant from the National Science Foundation (1539068). C.K. acknowledges a Royal College of Surgeons' Office of Research and Innovation Seed Fund Award (GR 14-0963), a Science Foundation Ireland (SFI) grant (SFI/12/RC/2278), and the European Union for a Marie Curie European Reintegration Grant under H2020 (Project Reference 659715). H.S. acknowledges the Fulbright Program.

\section{References}

[1] Peppas NA, Leobandung W. Stimuli-responsive hydrogels: Ideal carriers for chronobiology and chronotherapy. J Biomater Sci Polym Ed 2004;15(2):125-44.

[2] Mormont MC, Levi F. Cancer chronotherapy: Principles, applications, and perspectives. Cancer 2003;97(1):155-69.

[3] Richardson TP, Peters MC, Ennett AB, Mooney DJ. Polymeric system for dual growth factor delivery. Nat Biotechnol 2001;19:1029-34.

[4] Brudno Y, Ennett-Shepard AB, Chen RR, Aizenberg M, Mooney DJ. Enhancing microvascular formation and vessel maturation through temporal control over multiple pro-angiogenic and pro-maturation factors. Biomaterials 2013;31:9201-9.

[5] Mehta M, Schmidt-Bleek K, Duda GN, Mooney DJ. Biomaterial delivery of morphogens to mimic the natural healing cascade in bone. Adv Drug Deliv Rev 2012;64(12):1257-76.

[6] Cao Y, Langer R. Optimizing the delivery of cancer drugs that block angiogenesis. Sci Trans Med 2010;2:15ps3.

[7] Kearney CJ, Mooney DJ. Macroscale delivery systems for molecular and cellular payloads. Nat Mater 2013;12:1004-17.

[8] Hoare TR, Kohane DS. Hydrogels in drug delivery: Progress and challenges. Polymer 2008;49(8):1993-07.

[9] Ron ES, Bromberg LE. Temperature-responsive gels and thermogelling polymer matrices for protein and peptide delivery. Adv Drug Deliv Rev 1998;31(3):197-221.

[10] Hoare T, Santamaria J, Goya GF, Irusta S. Lin D, Lau S, Padera R, Langer R, Kohane DS. A magnetically triggered composite membrane for on-demand drug delivery. Nano Lett 2009;9(10):3651-1.

[11]Zhao X, Kim J, Cezar CA, Huebsch N, Lee K, Bouhadir K, Mooney DJ. Active scaffolds for on-demand drug and cell delivery. Proc Natl Acad Sci USA 2010;108:67-72.

[12] Cezar CA, Kennedy SM, Mehta M, Weaver JC, Vandenburgh H, Mooney DJ. Biphasic ferrogels for triggered drug and cell delivery. Adv Healthc Mater 2014;3(11):1869-76.

[13] Nair M, Guduru R, Liang P, Hong J, Sagar V, Khizroev S. Externally controlled on-demand release of antiHIV drug using magneto-electric nanoparticles as carriers. Nat Commun 2013;4:1707.

[14] Kennedy SM, Bencherif S, Norton D, Weinstock L, Mehta M, Mooney DJ. Rapid and extensive collapse from electrically responsive macroporous hydrogels. Adv Healthc Mater 2014;3(4):500-7.

[15] Alvarez-Lorenzo C, Bromberg L, Concheiro A. Light-sensitive intelligent drug delivery systems. Photochem Photobiol 2009;85(4):848-60.

[16] Farra R, Sheppard NF, McCabe L, Neer RM, Anderson JM, Santini JT, Cima MJ, Langer R. First-in-human testing of a wirelessly controlled drug delivery microchip. Sci Transl Med 2012;4:122ra21.

[17] Mitragotri S. Healing sound: The use of ultrasound in drug delivery and other therapeutic applications. Nat Rev Drug Discov 2005;4(3):255-60.

[18] Brown MR, Farguhar-Smith P, Williams JE, Ter Haar G, deSouza NM. The use of high-intensity focused ultrasound as a novel treatment for painful conditions - a description and narrative of the literature. $\mathrm{Br} \mathrm{J}$ Anaesth 2015;115(4):520-30.

[19] Huebsch N, Kearney CJ, Zhao X, Kim J, Cezar CA, Suo Z, Mooney DJ. Ultrasound-triggered disruption and self-healing of reversibly cross-linked hydrogels for drug delivery and enhanced chemotherapy. Proc Natl Acad Sci USA 2014;111(27):9762-7. 
[20] Epstein-Barash H, Orbey G, Polat BE, Ewoldt RH, Feshitan J, Langer R, Borden MA, Kohane DS. A microcomposite hydrogel for repeated on-demand ultrasound-triggered drug delivery. Biomaterials 2010;31(19):5208-17.

[21] Kost J, Leong K, Langer R. Ultrasound-enhanced polymer degradation and release of incorporated substances. Proc Natl Acad Sci USA 1989;86(20):7663-6.

[22] Kearney CJ, Skaat H, Kennedy SM, Hu J, Darnell, M, Raimondo RM, Mooney DJ. Switchable release of entrapped nanoparticles from alginate hydrogels. Adv Healthc Mater 2015; E-pub ahead of print.

[23] Betz OB, Betz VM, Nazarian A, Egermann M, Gerstenfeld LC, Einhorn TA, Vrahas S, Bouxsein ML, Evans $\mathrm{CH}$. Delayed administration of adenoviral BMP-2 vector improves the formation of bone in osseous defects. Gene Ther 2007;14:1039-44.

[24] Bertone AL, Pittmann DD, Bouxsein M, Li J, Clancy B, Seeherman H. Adenoviral-mediated transfer of hBMP6 gene accelerates osteotomy repair and return of bone mechanical properties. Trans Orth Res Soc 2002;27:0279.

[25] Ito T, Tokunaga K, Maruyama H, Kawashima H, Kitahara H, Horikoshi T, Ogose A, Hotta Y, Kuwano R, Katagirl H, Endo N. Coxsackievirus and adenovirus receptor (CAR)-positive immature osteoblasts as targets of adenovirus-mediated gene transfer for fracture healing. Gene Ther 2003;10:1623-8.

[26] Goldman A, Majumder B, Dhawan A, Ravi S, Goldman D, Kohandel M, Majumder PK, Sengupta S. Temporally sequenced anticancer drugs overcome adaptive resistance by targeting a vulnerable chemotherapyinduced phenotypic transition. Nat Commun 2015:6:6139.

[27] Xiao D, Yang D, Guo L, Lu W, Charpentier M, Yan B. Regulation of carboxylaesterase-2 expression by p53 family proteins and enhanced anti-cancer activities among 5-fluorouracil, irinotecan and doxazolidine prodrug. Br J Pharmacol 2013;168(8):1989-99.

[28] Boontheekul T, Kong HJ, Mooney DJ. Controlling alginate gel degradation utilizing partial oxidation and bimodal molecular weight distribution. Biomaterials 2005;26(15):2455-65.

[29] Kim J, Cao D, Shvartsman E, Silva A, Mooney DJ. Targeted delivery of nanoparticles to ischemic muscle for imaging and therapeutic angiogenesis. Nano Lett 2011;11(2):694-700.

[30] Carragee EJ, Hurwitz EL, Weiner BK. A critical review of recombinant human bone morphogenetic protein-2 trials in spinal injury surgery: emerging safety concerns and lessons learned. Spine J 2011;11(6):471-91.

[31] Noble ML, Mourand PD, Ranter BD. Digital drug delivery: on-off ultrasound controlled antibiotic release from coated matrices with negligible background leaching. Biomater Sci 2014;2:893-902.

[32] Lattin JR, Pitt WG. Factors affecting ultrasonic release from eLiposomes. J Pharm Sci 2015;104:1373-84.

[33] Liang B, Tong R, Want Z, Guo S, Xia H. High intensity focused ultrasound responsive metallo-supramolecular block copolymer micells. Langmuir 2014;30(31):9524-32.

[34] Huang X, Brazel CS. On the importance and mechanisms of burst release in matrix-controlled drug delivery systems. J Control Release 2001;73(2):121-36.

[35] Kost J, Leong K, Langer R. Ultrasound-enhanced polymer degradation and release of incorporated substances. Proc Natl Acad Sci USA 1989;86(20):7663-6.

[36]Zhang L, Salsac A-V. Can sonication enhance release from liquid-core capsules with a hydrogel membrane? J Colloid Interface Sci 2012;368:648-54.

[37] Huang S-L, MacDonald RC. Acoustically active liposomes for drug encapsulation and ultrasound-triggered release. Biochimica et Biophysica Acta 2004;1665:134-41.

[38] Schroeder A, Honen R, Turjeman K, Gabizon A, Kost J, Barenholz Y. Ultrasound triggered release of cisplatin form liposomes in murine tumors. J Control Release 2009;137:63-68.

[39] Florinas S, Kim J, Nam K, Janát-Amsbury MM, Kim SW. Ultrasound-assisted siRNA delivery via argininegrafted bioreducible polymer and microbubble targeting VEGF for ovarian cancer treatments. J Control Release 2014;183:1-8.

[40] Sun RR, Noble ML, Sun SS, Song S, Miao CH. Development of therapeutic microbbubles for enhancing ultrasound-mediated gene delivery. J Control Release 2014;182:111-20.

[41] Wee S, Gombotz WR. Protein release from alginate matrices. Adv Drug Deliv Rev 1998;31(3):267-85.

[42] Cao Y, Wang B, Wang Y, Lou D. Dual release from core-shell nanoparticles with distinct release profiles. J Pharm Sci 2014;103(10):3205-16.

[43] Abbaspourrad A, Caroll NJ, Kim S-H, Weitz DA. Polymer microcapsules with programmable active release. J Am Chem Soc 2013;135(20):7744-50.

[44] Esser-Kahn AP, Sottos NR, White SR, Moore JS. Programmable microcapsules from self-immolative polymers. J Am Chem Soc 2010;132:10266-8. 
[45] Shi J, Votruba AR, Farokhzad OC, Langer R. Nanotechnology in drug delivery and tissue engineering: from discovery to applications. Nano Lett 2010;10(9):3223-30.

[46] Powell AC, Paciotti GF, Libutti SK. Colloidal gold: a novel nanoparticle for targeted cancer therapeutics. Methods Mol Biol 2010;624:375-84.

[47] Murphy CJ, Gole AM, Stone JW, Sisco PN, Alkilany AM, microcapsules Goldsmith EC, Baxter SC. Gold nanoparticles in biology: beyond toxicity to cellular imaging. Acc Chem Res 2008;41(12):1721-30.

[48] Hakkinen H. The goldsulfur interface at the nanoscale. Nat Chem 2012;4(6):443-55.

[49] Yi C, Liu D, Fong CC, Zhang J, Yang M. Gold nanoparticles promote osteogenic differentiation of mesenchymal stem cells through p38 MAPK pathway. ACS Nano 2010;4(11):6439-48.

[50] Hubbel JA. Biomaterials in tissue engineering. Nat Biotechnol 1995;13:565-76.

[51] Takabayashi T, Sato S, Sato A, Ozawa N, Sou S, Yajima A, Suzuki M. Influence of puls-wave ultrasonic irradiation on the prenatal development of mouse. Tohoku J Exp Med 1985;147(4):403-10.

[52] Hynyen K, McDannold N, Martin H, Jolesz FA, Vykodtseva N. The threshold for brain damage in rabbits induced by bursts of ultrasound in the presence of an ultrasound contrast agent. Ultrasound Med Biol 2003;29(3):473-81.

[53] Phenix CP, Togtema M, Pichardo S, Zehbe I, Curiel L. High intensity focused ultrasound technology, its scope and applications in therapy and drug delivery. J Pharm Pharm Sci 2014;17(1):136-53.

[54] Bezagu M, Errico C, Chaulot-Talmon V, Monti F, Tanter M, Tabling P, Cossy J, Areniyadis S, Couture O. High spatiotemporal control of spontaneous reactions using ultrasound-triggered composite droplets. J Am Chem Soc 2014;136:7205-8.

[55] Varde NK, Pack DW. Microspheres for controlled release drug delivery. Expert Opin Drug Biol Ther 2004;4:35-51.

[56] Cleland JL. Single-administration vaccines: controlled-release technology to mimic repeated immunizations. Tends Biotech 1999;17:25-29.

[57] Shi L, Caulfield MJ. Chern RT, Wilson RA, Gautam S, Bolkin DB. Pharmaceutical and immunological evaluation of a single-shot hepatitis B vaccine formulated with PLGA microspheres. J Pharm Sci 2002;91:10191035 .

[58] Cleland JL, Lim A, Daugherty A, et al. Development of a single-shot subunit vaccine for HIV-1 with programmable in vivo autoboost and long-lasting neutralization response. J Pharm Sci 1998;87:1489-1495.

[59] Sanchez A, Gupta RK, Alonso MJ, Siber GR, Langer R. Pulsed controlled-release system in vaccine delivery. J Pharm Sci 1996;85:547-552 